\title{
Discovering baryon-number violating neutralino decays at the LHC
}

\author{
Jonathan M. Butterworth, ${ }^{1}$ John R. Ellis, ${ }^{2}$ Are R. Raklev,${ }^{3}$ and Gavin P. Salam ${ }^{4}$ \\ ${ }^{1}$ Department of Physics 85 Astronomy, University College London, UK \\ ${ }^{2}$ Theory Division, Physics Department, CERN, CH-1211 Geneva 23, Switzerland \\ ${ }^{3}$ DAMTP, University of Cambridge, Cambridge CB3 OWA, UK; \\ Cavendish Laboratory, University of Cambridge, Cambridge CB3 OHE, UK \\ ${ }^{4}$ LPTHE, UPMC Univ. Paris 6, CNRS UMR 7589, Paris, France
}

\begin{abstract}
Recently there has been much interest in the use of single-jet mass and jet substructure to identify boosted particles decaying hadronically at the LHC. We develop these ideas to address the challenging case of a neutralino decaying to three quarks in models with baryonic violation of R-parity. These decays have previously been found to be swamped by QCD backgrounds. We demonstrate for the first time that such a decay might be observed directly at the LHC with high significance, by exploiting characteristics of the scales at which its composite jet breaks up into subjets.
\end{abstract}

The LHC's scope for the discovery of supersymmetry broken at the TeV scale [1 5] has generated much interest. Certainly, the potential prize is great: $\mathrm{TeV}$-scale supersymmetry could solve several puzzling problems and answer a number of open questions in modern particle physics, such as the fine-tuning of the Higgs mass, the unification of forces at high energies and the nature of dark matter. Effort has mainly been concentrated on investigations into the discovery reach and possibility of parameter measurements in the Minimal Supersymmetric Standard Model (MSSM) and various more constrained versions featuring a weakly-interacting and stable Lightest Supersymmetric Particle (LSP), which gives a missing-energy signature. Candidates for the LSP include the lightest neutralino, $\tilde{\chi}_{1}^{0}$, and the gravitino.

However, the gauge symmetries of the Standard Model (SM) also allow for dimension-four terms in the superpotential of the forms

$$
\lambda_{i j k} L_{i} L_{j} \bar{E}_{k}+\lambda_{i j k}^{\prime} L_{i} Q_{j} \bar{D}_{k}+\lambda_{i j k}^{\prime \prime} \bar{U}_{i} \bar{D}_{j} \bar{D}_{k}
$$

which violate the R-parity that is imposed in the MSSM. Non-zero values for the couplings $\lambda$ could imply drastically different phenomenologies for supersymmetry at the LHC, allowing for the decay of the LSP, or any heavier sparticle, directly to SM particles.

Broadly speaking, we can classify the RPV models in terms of the dominant coupling $\lambda$ and the identity of the Next-to-Lightest Supersymmetric Particle (NLSP), assuming that a gravitino is the LSP. The lepton-numberviolating (LV) couplings $\lambda$ and $\lambda^{\prime}$ generally give MSSMlike signatures with missing energy from neutrinos and/or extra leptons in the decays of the NLSP. The exception is a slepton/sneutrino NLSP that decays into two quarks via a dominant $\lambda^{\prime}$ coupling. Scenarios such as these should be easy to extract from the SM backgrounds, as shown for a neutralino NLSP in [6].

However, dominant baryon-number-violating (BV) couplings $\lambda^{\prime \prime}$ are more difficult to deal with, due to the large hadronic activity expected at the LHC, which threatens to drown decays such as $\tilde{\chi}_{1}^{0} \rightarrow q q q$. The QCD background for jets with $p_{T}>500 \mathrm{GeV}$ and a $\tilde{\chi}_{1}^{0}$ mass of $\mathcal{O}(100 \mathrm{GeV})$ is about two orders of magnitude higher than the signal. The background's non-trivial shape means that it is hard to establish whether a small deviation from the expected background is a signal of something new, or simply a defect in one's understanding of the background. Some success has been reported by relying on the production of a high- $p_{T}$ lepton in the decay chain leading to the NLSP [6-8], but ideally one would wish to demonstrate the feasibility of signal isolation and mass measurement in a less model-dependent manner. Otherwise, the fear is that supersymmetry could escape discovery at the LHC by cloaking itself in BV decays.

In this Letter we investigate that problem by looking for jets from the decays of very boosted sparticles via BV couplings. Such decays give rise to composite jets made up of two or more collimated subjets, with a jet mass related to that of the original sparticle, with specific properties predicted for the scale at which the main jet separates into subjets. Similar techniques have previously been used by the authors for analysing $W W$ scattering [9, 10], for detecting massive boson decays in MSSM scenarios [11] and in Higgs searches [12]. In addition, a number of other techniques for separating hadronic decays of heavy particles from QCD backgrounds have been suggested by other groups 13-19]. While some of these studies are applicable to general searches, they all worked with examples of particles with known or wellconstrained mass. The present article tackles a new problem: how to identify a hadronic resonance of unknown mass in an explicitly scale-invariant manner. The techniques presented here in a supersymmetric scenario with RPV clearly have applications to any hadronicallydecaying massive-particle resonance that can be produced far above threshold, and are promising for broad use in the challenging LHC searches for hadronic decays of new particles.

We focus our investigation on the CMSSM benchmark point SPS1a. We also look at six other CMSSM points with larger sparticle masses and lower cross sections, that lie along the corresponding benchmark line [20]. These encompass sparticle masses from $\left(m_{\tilde{\chi}_{1}^{0}}, m_{\tilde{q}}, m_{\tilde{g}}\right) \simeq$ $(96,530,600) \mathrm{GeV}$ to $(161,815,915) \mathrm{GeV}$, and cross sections from $47 \mathrm{pb}$ to $4.6 \mathrm{pb}$ at leading order. We note that 
the gluino and squark masses are much larger than the neutralino mass, and can hence yield a highly-boosted neutralino in their decays.

$\mathrm{R}$-parity violation is incorporated by setting the dominant coupling to be $\lambda_{112}^{\prime \prime}=0.001$. This coupling is chosen to be difficult: no heavy flavours are present to help tag the correct jets and the coupling is chosen to be relatively large, so that decays do not lead to displaced vertices [21].

In order to simulate sparticle pair-production events at the LHC with RPV decays, we use the Herwig 6.510 Monte Carlo event generator 22 25] with CTEQ 6L [26] PDFs, and use JimmY 4.31 [27] for the simulation of multiple interactions 28]. This is interfaced to the FASTJET 2.4.0 [29, 30] jet-finder package using the RIVET [31] framework. Our background sample, consisting of QCD $n$-jet events, $t \bar{t}, W+$ jet, $Z+$ jet and $W W / W Z / Z Z$ production is simulated with the same setup, with the exception of the $n$-jet events where we use Alpgen 2.13 [32] interfaced to HERWIG/JIMMY to generate up to 4-jet final states with the MLM jet-parton matching scheme. For the QCD event generation we also require $\sum p_{T}>$ $600 \mathrm{GeV}$ to reduce the large sample size, where the sum is over all jets. The leading-logarithmic parton shower approximation that is used has been shown to model jet substructure well in a wide variety of processes [33 38 .

For both signal and background we generate a number of events equivalent to $1 \mathrm{fb}^{-1}$ of LHC data at $14 \mathrm{TeV}$ CM energy. No attempt is made at detector simulation through finite calorimeter granularity, but we do impose a geometrical acceptance cut on jets of $|\eta|<2.5$.

To illustrate the variety of approaches that can be taken with subjet studies, we consider two complementary analyses: the first is an exclusive analysis with the $k_{T}$ algorithm [39, 40] and searches for substructure in two jets, one for each neutralino expected in an event. The other is an inclusive analysis, with the Cambridge/Aachen (C/A) algorithm [41, 42], which examines the substructure of just the hardest jet. We find that the corresponding inclusive (exclusive) analysis with the $k_{T}$ (C/A) algorithm give similar results.

The $k_{T}$ algorithm defines distances $d_{k l} \equiv$ $\min \left(p_{T k}^{2}, p_{T l}^{2}\right)\left(\Delta R_{k l}^{2} / R^{2}\right), d_{k B} \equiv p_{T k}^{2}$, and sequentially merges the pair of objects $k, l$ with smallest $d_{k l}$, unless there is a smaller $d_{k B}$, in which case $k$ becomes a jet. The constant $R$ sets the angular reach of the jets. Since the $k_{T}$ distance is just the relative transverse momentum between objects, the mergers of interest for a decayed heavy-particle tend to be the last ones. This was exploited in [9 11], where a dimensionful cut was placed on the $d_{k l}$ scale of the last merging in the jet, $d_{1}$, in order to preferentially select boosted $W$ bosons over QCD jets, which, for a given mass, have smaller $d_{1}$.

However, our case differs from [9 11] in two respects. First, we are searching for an object of unknown mass, which means that we should avoid biasing the search with a dimensionful substructure cut. A good alternative is to cut on a dimensionless variable normalised to the jet mass $m_{j}, y_{i}=d_{i} R^{2} / m_{j}^{2}$. Secondly, the neutralino has a three-
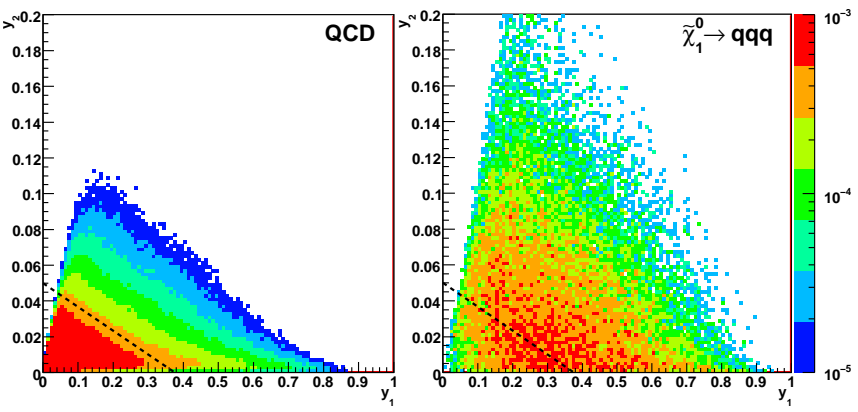

FIG. 1: The $y$-values from the $k_{T}$ algorithm with $R=0.4$ for the last and next-to-last merging for QCD jets (left), and jets matched to neutralinos (right). Also shown is the proposed cut line. Both distributions are normalized to unity.

body decay, in contrast to a W-boson's two-body decay. This suggests that one should cut on the properties of the last two mergers, i.e. on $y_{1}$ and $y_{2}$. Their distributions are illustrated in Fig. 1. The left panel shows QCD jets with $p_{T}>300 \mathrm{GeV}$, while the right panel shows jets matched to neutralinos for the SPS1a benchmark point, the jet being within $\Delta R<0.3$ of the neutralino with $p_{T}>300 \mathrm{GeV}$ and mass $90 \mathrm{GeV}<m_{j}<120 \mathrm{GeV}$. Even after the hard cut on jet $p_{T}$, there are clear differences between neutralino jets and the QCD background.

For our analysis with the $k_{T}$ algorithm, we take $R=$ 0.4 and use the following two cuts: i) at least four jets with $p_{T}>300,300,100,100 \mathrm{GeV}$ and two of the high- $p_{T}$ jets $\left(p_{T}>300 \mathrm{GeV}\right)$ should have significant substructure, $y_{2}>-0.13 y_{1}+0.05$, consistent with fully or partially contained neutralinos. The choice of $R$ is a compromise between capturing sufficient signal, favouring large $R$, and not smearing the mass peak with particles from the underlying event, favouring small $R$ 43]. Cuts on a third and fourth jet are motivated by the expected presence of two jets from the squark or gluino decay. We have verified that our simulation of QCD events with additional jets is consistent with NLO calculations in [44]. The requirement of high $p_{T}$ should ensure high trigger efficiency, as well as good collimation of the neutralino jets.

The mass distribution for the neutralino candidate jets after cuts is shown in Fig. 2 (left). The $\tilde{\chi}_{1}^{0}$ is clearly visible as a perturbation on the rapidly falling QCD background. This analysis reconstructs $3.3 \%$ of all neutralinos with $p_{T}>300 \mathrm{GeV}$ (constituting $17.8 \%$ of neutralinos produced) in a $20 \mathrm{GeV}$ mass window around the nominal mass; most reconstructed neutralinos have a $p_{T}$ somewhat above the $300 \mathrm{GeV}$ cut. For the QCD jets the analysis accepts $0.023 \%$ of all jets with $p_{T}>300 \mathrm{GeV}$ in the same window.

Even though we have introduced only dimensionless jet substructure cuts in the above analysis, the background distribution also has a peak near the neutralino mass. This is a consequence of higher-order perturbative effects [17, 45] and the peak position is determined by 

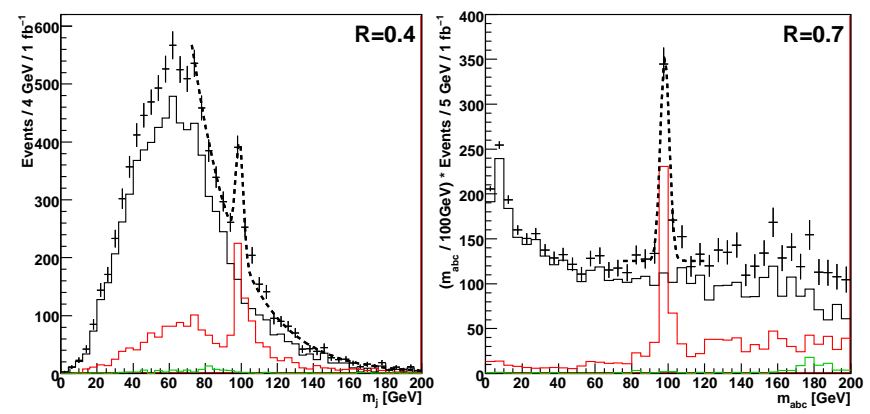

FIG. 2: Jet mass distribution (points with error bars) for the $k_{T}$ algorithm (left) and $\mathrm{C} / \mathrm{A}$ algorithm (right) after all cuts. Also shown are the contributions from QCD (black), supersymmetric events (red) and other SM backgrounds (green).

their interplay with $R$, the jet $p_{T}$ and substructure cuts. Increasing $R$, which improves the signal reconstruction efficiency, causes the background peak to move to larger mass, potentially directly beneath the signal peak

To avoid this issue, we consider the $\mathrm{C} / \mathrm{A}$ algorithm, which successively recombines the pair of objects closest in $\Delta R_{k l}$, until all objects are separated by more than $R$, at which point they are the jets. Because the ordering of $\mathrm{C} / \mathrm{A}$ mergers knows nothing about the momentum scales involved, one cannot rely on the properties of the last mergers to tag relevant substructure.

Instead we recurse through all mergers storing those subjets that are sufficiently symmetric, having $z \equiv$ $\min \left(p_{T k}, p_{T l}\right) /\left(p_{T k}+p_{T l}\right)>z_{\min }$, ignoring in the recursion the softer of the two subjets when $z<z_{\min }$. From this we identify the two mergers that have the largest JADE-type distance, $d_{k l}^{J}=p_{T k} p_{T l} \Delta R_{k l}^{2}$, which is related to $m_{k l}^{2}$ if $k$ and $l$ are massless. If the subjet resulting from the merger with smaller $d^{J}$ (labeling this subjet "bc") is contained within the subjet from the other merger (labeled "abc"), i.e. the subjet "abc" is the result of further mergers of "bc", then we consider "abc" to be a neutralino candidate if $\mu \equiv m_{b c} / m_{a b c}>\mu_{\min }$. The cut on $z$ is used to avoid the soft splittings that dominate QCD branching and that are largely responsible for producing the peak in the mass distribution of QCD jets. The cut on the subjet mass ratio $\mu$ ensures the presence of a three-body decay structure inside the jet.

The full C/A-based analysis proceeds as follows: i) we use $R=0.7$ and require at least four jets with $p_{T}>500,100,100,80 \mathrm{GeV}$, and $\left|\Delta \eta_{13}\right|,\left|\Delta \eta_{14}\right|<1.5$, ii) the hardest jet is taken to be a neutralino candidate if it passes the substructure cuts with $z_{\min }=0.15$ and $\mu_{\text {min }}=0.25$. Relative to the $k_{T}$ analysis, the harder jet cuts help keep the background under control despite requiring substructure in only one jet. For events that pass the cuts, we plot in Fig. 2 (right) the distribution of $m_{a b c}$, weighted with $m_{a b c} / 100 \mathrm{GeV}$. Expectations from QCD are that this distribution should be rather flat for $m_{\min } \lesssim m_{j} \lesssim p_{T} R \sqrt{z_{\min }}$, where $m_{\text {min }}$ is some small value governed by higher orders. This is indeed
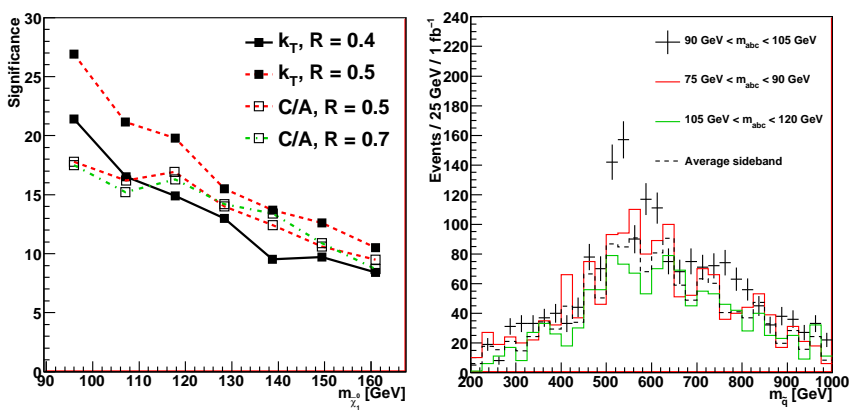

FIG. 3: Estimated sensitivity for $1 \mathrm{fb}^{-1}$ as a function of $\tilde{\chi}_{1}^{0}$ mass for various choices of jet algorithm and size $R$ (left), jet mass distribution for a squark search using the $\mathrm{C} / \mathrm{A}$ algorithm with $R=0.5$ and background estimation by sidebands (right).

what we observe, and for a range of choices of $R$ value and $p_{T}$ cut the signal is found to lie in this interval. The analysis reconstructs $10.3 \%$ of all neutralinos with $p_{T}>500 \mathrm{GeV}$ (constituting $3.7 \%$ of all neutralinos produced) in a $20 \mathrm{GeV}$ mass window around the nominal mass; for QCD jets $0.052 \%$ of all jets with $p_{T}>500 \mathrm{GeV}$ are accepted in this mass window.

For both analyses we estimate the significance of the signal from the number of signal and background events in a window of $20 \mathrm{GeV}$ centered on the peak, a choice consistent with the $7 \%$ mass resolution seen in ATLAS detector simulations [10]. This ignores the effect of the 'looking-elsewere' problem, but should demonstrate the potential of such a search. The results are shown in Fig. 3 (left) for various neutralino masses along the SPS1a benchmark line. Even in the highest-mass case studied, the significance is well above the $5-\sigma$ discovery 'threshold' with $1 \mathrm{fb}^{-1}$ of statistics. In fact, the order of magnitude loss in cross section over the parameter range is to a large extent compensated by the lower QCD background at higher jet masses for the $k_{T}$ analysis, and in the $\mathrm{C} / \mathrm{A}$ case, jets from higher-mass squark and gluino decays are more likely to pass our hard $p_{T}$ cut.

With evidence for a resonance peak, the next step is to estimate the mass of the resonance. We fit the jet-mass distributions with a background plus Gaussian signal distribution. The $k_{T}$ analysis uses an exponential background in the interval $[80,200] \mathrm{GeV}$, while the $\mathrm{C} / \mathrm{A}$ analysis uses a uniform background in the interval $[75,120] \mathrm{GeV}$. The results of this naive fit, which ignores the experimental jet mass resolution, are shown in Table [ for the SPS1a benchmark point. Improvements on the systematic errors inherent in this method are possible by calibrating the jet mass against the known masses of the $W$ boson or top quark, for which a reasonably clean measurement should be possible in events in which one top quark decays leptonically. Improvements in mass measurement are also possible through filtering of jets [12].

In Fig. 3 (right) we also demonstrate the potential of our method for reconstructing the squark mass. By se- 


\begin{tabular}{|l|c|c|c|c|}
\hline Analysis & $m_{\tilde{\chi}_{1}^{0}}$ & $\chi^{2} / \mathrm{ndf}$ & $m_{\tilde{q}_{R}}$ & $\chi^{2} / \mathrm{ndf}$ \\
\hline$k_{T}, \mathrm{R}=0.4$ & $99.0 \pm 0.5$ & 1.43 & - & - \\
\hline $\mathrm{C} / \mathrm{A}, \mathrm{R}=0.5$ & $98.2 \pm 0.4$ & 0.85 & $517.0 \pm 2.5$ & 1.27 \\
\hline $\mathrm{C} / \mathrm{A}, \mathrm{R}=0.7$ & $98.4 \pm 0.6$ & 1.46 & $526.6 \pm 3.0$ & 1.20 \\
\hline
\end{tabular}

TABLE I: Neutralino and squark mass fits for SPS1a. The nominal masses are $m_{\tilde{\chi}_{1}^{0}}=96.1 \mathrm{GeV}$ and $m_{\tilde{q}_{R}}=520 \mathrm{GeV}$.

lecting events from the $\mathrm{C} / \mathrm{A}$ analysis in the signal band $90 \mathrm{GeV}<m_{a b c}<105 \mathrm{GeV}$ and plotting both the mass of the neutralino combined with the third jet and combined with the fourth jet in the event, we arrive at the distribution in black, with a clear peak around $520 \mathrm{GeV}$. The interpretation of the peak is checked by plotting the sideband distributions, picking events from $75 \mathrm{GeV}<$ $m_{a b c}<90 \mathrm{GeV}$ (red) and $105 \mathrm{GeV}<m_{a b c}<120 \mathrm{GeV}$ (green). These show no sign of a peak. By subtracting the averaged sidebands (dashed line), and fitting the remaining peak with a Gaussian, we arrive at the squark mass estimates in Table I]

The effects of pile-up, intrinsic resolution and granularity of the detector will all have additional impact on the discovery of a neutralino resonance and the measurement of its mass at the LHC, but initial studies with realistic detector simulations indicate that the efficiencies and resolutions assumed here are not unreasonable [10]. Further studies of the techniques presented here are ongoing within the ATLAS collaboration [46].

In conclusion, we see that using sophisticated jet clustering algorithms such as $k_{T}$ and $\mathrm{C} / \mathrm{A}$ gives us the possibility of discovering baryon-number violating decays of the type $\tilde{\chi}_{1}^{0} \rightarrow q q q$, without the assumption of additonal features such as hard leptons, and even when using only the substructure of the hardest jet in the event. We have further found that the neutralino mass can be measured to a precision of a few $\mathrm{GeV}$ in these R-parity-violating scenarios, most likely limited by the experimental jet mass resolution, and that one can identify the squark resonance. Realizing the potential outlined in the above analyses is a challenge that merits experimental study.

This work was supported by the UK Science and Technology Facilities Council (STFC). ARR whishes to thank the members of the Cambridge Supersymmetry Working Group for many useful discussions. GPS thanks Matteo Cacciari and Gregory Soyez for collaboration on FastJet development and Pietro Slavich for helpful comments.
[1] J. Wess and B. Zumino, Nucl. Phys. B70, 39 (1974).

[2] P. Fayet and S. Ferrara, Phys. Rept. 32, 249 (1977).

[3] S. Dimopoulos and H. Georgi, Nucl. Phys. B193, 150 (1981).

[4] H. P. Nilles, Phys. Rept. 110, 1 (1984).

[5] H. E. Haber and G. L. Kane, Phys. Rept. 117, 75 (1985).

[6] ATLAS Collaboration, I. Hinchliffe et al., CERN-LHCC99-15.

[7] B. C. Allanach et al., JHEP 03, 048 (2001), [hepph/0102173].

[8] B. C. Allanach et al., JHEP 09, 021 (2001), [hep$\mathrm{ph} / 0106304]$.

[9] J. M. Butterworth, B. E. Cox and J. R. Forshaw, Phys. Rev. D65, 096014 (2002), [hep-ph/0201098].

[10] ATLAS Collaboration, G. Aad et al., arXiv:0901.0512.

[11] J. M. Butterworth, J. R. Ellis and A. R. Raklev, JHEP 05, 033 (2007), [hep-ph/0702150].

[12] J. M. Butterworth, A. R. Davison, M. Rubin and G. P. Salam, Phys. Rev. Lett. 100, 242001 (2008), [arXiv:0802.2470].

[13] K. Agashe et al., Phys. Rev. D77, 015003 (2008), [hep$\mathrm{ph} / 0612015]$.

[14] G. H. Brooijmans et al., arXiv:0802.3715 [hep-ph].

[15] D. E. Kaplan, K. Rehermann, M. D. Schwartz and B. Tweedie, Phys. Rev. Lett. 101, 142001 (2008), [arXiv:0806.0848].

[16] J. Thaler and L.-T. Wang, JHEP 07, 092 (2008), [arXiv:0806.0023].

[17] L. G. Almeida et al., arXiv:0807.0234.

[18] D. Krohn, J. Thaler and L.-T. Wang, arXiv:0903.0392.

[19] S. D. Ellis, C. K. Vermilion and J. R. Walsh, arXiv:0903.5081.

[20] B. C. Allanach et al., Eur. Phys. J. C25, 113 (2002), [hep-ph/0202233].

[21] We do not address the issue of constraints on this coupling, but note that our results are readily transferable to other couplings that are less restricted, such as $\lambda_{212}^{\prime \prime}$ [47].

[22] G. Corcella et al., JHEP 01, 010 (2001), [hep$\mathrm{ph} / 0011363]$.

[23] H. K. Dreiner, P. Richardson and M. H. Seymour, JHEP 04, 008 (2000), [hep-ph/9912407].

[24] S. Moretti et al., JHEP 04, 028 (2002), [hep$\mathrm{ph} / 0204123]$.

[25] G. Corcella et al., hep-ph/0210213.

[26] J. Pumplin et al., JHEP 07, 012 (2002), [hep$\mathrm{ph} / 0201195]$.

[27] J. M. Butterworth, J. R. Forshaw and M. H. Seymour, Z. Phys. C72, 637 (1996), [hep-ph/9601371].

[28] The non-default parameter settings for JIMMY are: $\mathrm{PRSOF}=0, \mathrm{PRRAD}=1.8, \mathrm{PTJIM}=4.9 \mathrm{GeV}, \mathrm{JMUEO}=1$, corresponding to an ATLAS tune [48].

[29] M. Cacciari and G. P. Salam, Phys. Lett. B641, 57 (2006), [hep-ph/0512210].

[30] M. Cacciari, G. P. Salam and G. Soyez, FastJet, http://fastjet.fr/

[31] B. M. Waugh et al., hep-ph/0605034.

[32] M. L. Mangano et al., JHEP 07, 001 (2003), [hep$\mathrm{ph} / 0206293]$.

[33] D0 Collaboration, V. M. Abazov et al., Phys. Rev. D65, 052008 (2002), [hep-ex/0108054].

[34] CDF Collaboration, D. E. Acosta et al., Phys. Rev. D71, 112002 (2005), [hep-ex/0505013].

[35] ZEUS Collaboration, S. Chekanov et al., Nucl. Phys. B700, 3 (2004), [hep-ex/0405065].

[36] OPAL Collaboration, G. Abbiendi et al., Eur. Phys. J. C37, 25 (2004), [hep-ex/0404026]. 
[37] OPAL Collaboration, G. Abbiendi et al., Eur. Phys. J. C31, 307 (2003), [hep-ex/0301013].

[38] ALEPH Collaboration, D. Buskulic et al., Phys. Lett. B384, 353 (1996).

[39] S. Catani, Y. L. Dokshitzer, M. H. Seymour and B. R. Webber, Nucl. Phys. B406, 187 (1993).

[40] S. D. Ellis and D. E. Soper, Phys. Rev. D48, 3160 (1993), [hep-ph/9305266].

[41] Y. L. Dokshitzer, G. D. Leder, S. Moretti and B. R. Webber, JHEP 08, 001 (1997), [hep-ph/9707323].

[42] M. Wobisch and T. Wengler, hep-ph/9907280.
[43] M. Dasgupta, L. Magnea and G. P. Salam, JHEP 02, 055 (2008), [0712.3014].

[44] Z. Nagy, Phys. Rev. Lett. 88, 122003 (2002), [hep$\mathrm{ph} / 0110315]$.

[45] S. Catani, L. Trentadue, G. Turnock and B. R. Webber, Nucl. Phys. B407, 3 (1993).

[46] ATLAS Collaboration, ATLAS-PHYS-PUB-2009-076.

[47] B. C. Allanach, A. Dedes and H. K. Dreiner, Phys. Rev. D60, 075014 (1999), [hep-ph/9906209].

[48] S. Alekhin et al., hep-ph/0601012. 